\title{
Geometrical Processing of 3D Body Scanner Data for Anthropometric Applications
}

\author{
Christian LOVATO ${ }^{a}$, Umberto CASTELLANI ${ }^{b}$, Carlo ZANCANARO ${ }^{a}$ and Andrea GIACHETTI ${ }^{\star b}$ \\ ${ }^{a}$ Dipartimento di Scienze Neurologiche, Neuropsicologiche, Morfologiche e Motorie \\ ${ }^{b}$ Dipartimento di Informatica, Università di Verona, Italy
}

\begin{abstract}
In this paper we describe a pipeline for the geometric processing of 3D meshes obtained by whole body scanners, aimed at providing support for anthropometric measurements.

The output of whole body scanners is a cloud of points, usually transformed in a triangulated mesh through the use of specific algorithms in order to support the 3D visualization of the surface and the extraction of meaningful anthropometric landmarks and measurements. Anthropometric analysis is usually performed by using device specific and closed software solutions provided by scanner manufacturers, and requires often a careful acquisition, with strong constraints on subject pose.

This may create problems in comparing data acquired in different places and performing large-scale multi-centric studies as well as in applying advanced shape analysis tools on the captured models.

The aim of our work is to overcome these problems by selecting and customizing geometrical processing tools able to create an open and device-independent pipeline for the analysis of body scanner data. We also aim at developing and validating methods to extract automatically feature points, body segments and relevant measurements that can be used in anthropometric and metabolic research.

The paper presents a general description of the proposed processing pipeline and the currently developed modules (hole filling, skeleton-based segmentation, heat kernel based landmark location), showing their usefulness in the automatic and computer assisted evaluation of anthropometric data.
\end{abstract}

Keywords: 3d body scanning, Digital Anthropometry, Mesh processing, Geometrical processing.

\section{3D body scanners based anthropometry and human body models quality}

An important application of 3D scanning of the human body is the use of the acquired model for anthropometric studies. Digital acquisition provide a large amount of data that been used to obtain automatically or interactively sets of measurements often applied in population surveys[1], also in relation to categorizing health risks [2].

Body scanner devices are usually equipped with software tools able process and export data. For anthropometric applications these tools include often the possibility to record sets of measurements characterizing the body shape and data acquired in this way have been recently used in validation studies and correlated with metabolic parameters $[3,4,5]$. A drawback of these measurements tools is that they are not standardized, parameters often are strongly dependent on the acquisition device and on pose [6], and there are not advanced tools to extract all the relevant medical information from the whole geometry.

In order to extract freely a posteriori all the interesting morphological information that could not only be collected for statistical studied, but could also be correlated, for example with body composition and metabolic parameters, it would be important to obtain high quality meshes from different kind of acquisition devices, and to specialize for these kind of meshes geometry processing techniques able to support automatic landmark location, skeletonization and segmentation of body parts.

In this paper we describe a series of tools for the analysis of three dimensional meshes that have been selected and customized for this purpose.

Our goal is to build a processing pipeline (see Fig. 1) that is device and pose independent and is designed in a hierarchical way, in the sense that, after a pre-processing step, we extract information at different levels of detail: in fact, we first recover, from a low-resolution, watertight version of the original mesh, a raw body representation, including a few evident anatomical landmarks, pose, segmentation of main parts (e.g. limbs, trunk, head/neck).

*andrea.giachetti@univr.it 


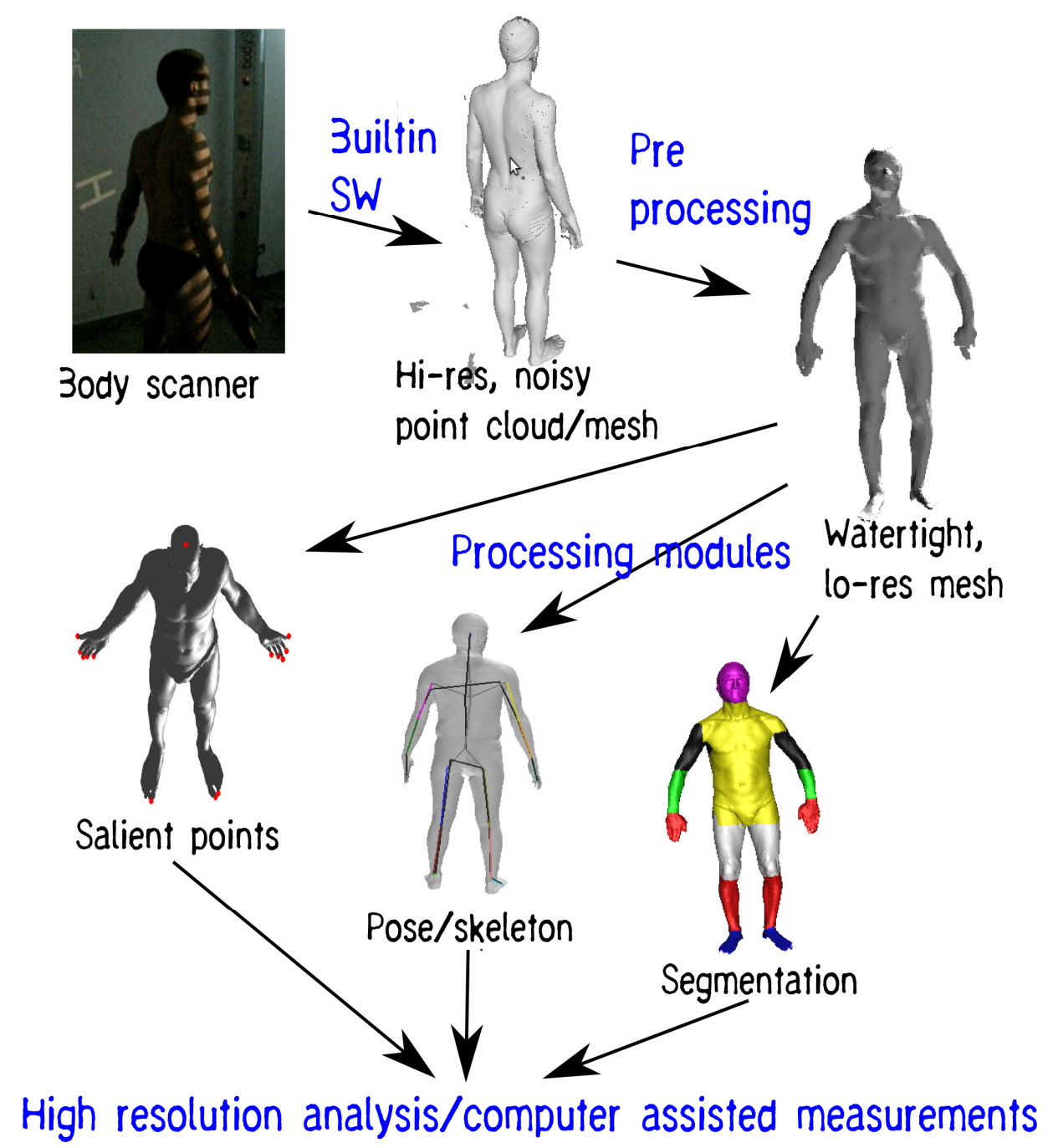

Figure 1: The whole body scanner processing pipeline. After the "raw" geometrical processing methods (described in the text), we plan to add a finer processing able to detect automatically anthropometric features and improving measurements precision.

This information can be used to obtain automatically local measurements, but also as an input for the subsequent "high resolution" processing, planned to refine the local geometry, to derive a context for the detection and segmentation of smaller structures on full resolution meshes with reduced computational effort. Currently the high resolution processing is limited to the development of interactive measurements methods, but the implementation of context aware high resolution feature recognition is planned for the next future.

The pipeline is currently made of different components, written both in $\mathrm{C}_{++}$and Matlab, we plan, however, when the tools will be sufficiently tested and validated, to include the different modules in an unique software framework, that should be the Meshlab processing system, developed by the Visual Computing Lab of ISTI - CNR with the support of the 3D-CoForm project $[17,18]$. We currently use this tool for mesh pre-processing and visualization and its software architecture allows an easy addition of new functionalities with the associated user interfaces by creating simple plug-ins.

The paper is organized as follows: Section 2 describes the pre-processing procedures necessary to create a "measurable" model, with particular focus on hole filling algorithms needed to overcome the weaknesses of the acquisition devices, Section 3 presents the "low-resolution" analysis including salient point extraction, skeletonization, segmentation and pose estimation, showing the results obtained, Section 4 shows preliminary results of high resolution processing and computer assisted measurements. 


\section{Pre-processing and hole filling}

Even if acquisition devices and related software packages are usually able to create and export triangulated meshes representing the scanned human body, the quality of these meshes is often not sufficient for the direct application of measurement algorithms providing volumes, geodesic distances, etc. Many mesh processing algorithms of our interest, in fact, requires input meshes that should be closed, without non-manifold faces, smooth. If we want then capture information at different levels of detail we should also be able to perform controlled mesh decimation and remeshing.

Even if all the pre-processing algorithms able to obtain these results are not always available in the software packages provided by body scanner manufacturers, many of them are included in the Meshlab distribution and are very easy to use. (e.g. removal of duplicated, unreferenced vertices, null faces, removal of small isolated components, coherent normal unification and flipping, erasing of non manifold faces, edge collapse simplification surface reconstruction from points with a ball pivoting variant, marching cubes and poisson's reconstruction).

The only procedure requiring particular attention and could not be performed with the available Meshlab methods is the filling of holes, that in Moirè-fringing-based scanners are related to the presence of reflective areas (e.g. hair), and that can be large, especially if the acquisition is not so careful. The procedure, is mandatory for our processing pipeline: shape analysis and measurement methods often require watertight meshes as input, and the accuracy of the reconstructed parts corresponding to original holes may relevantly affect the output of the subsequently applied algorithms.

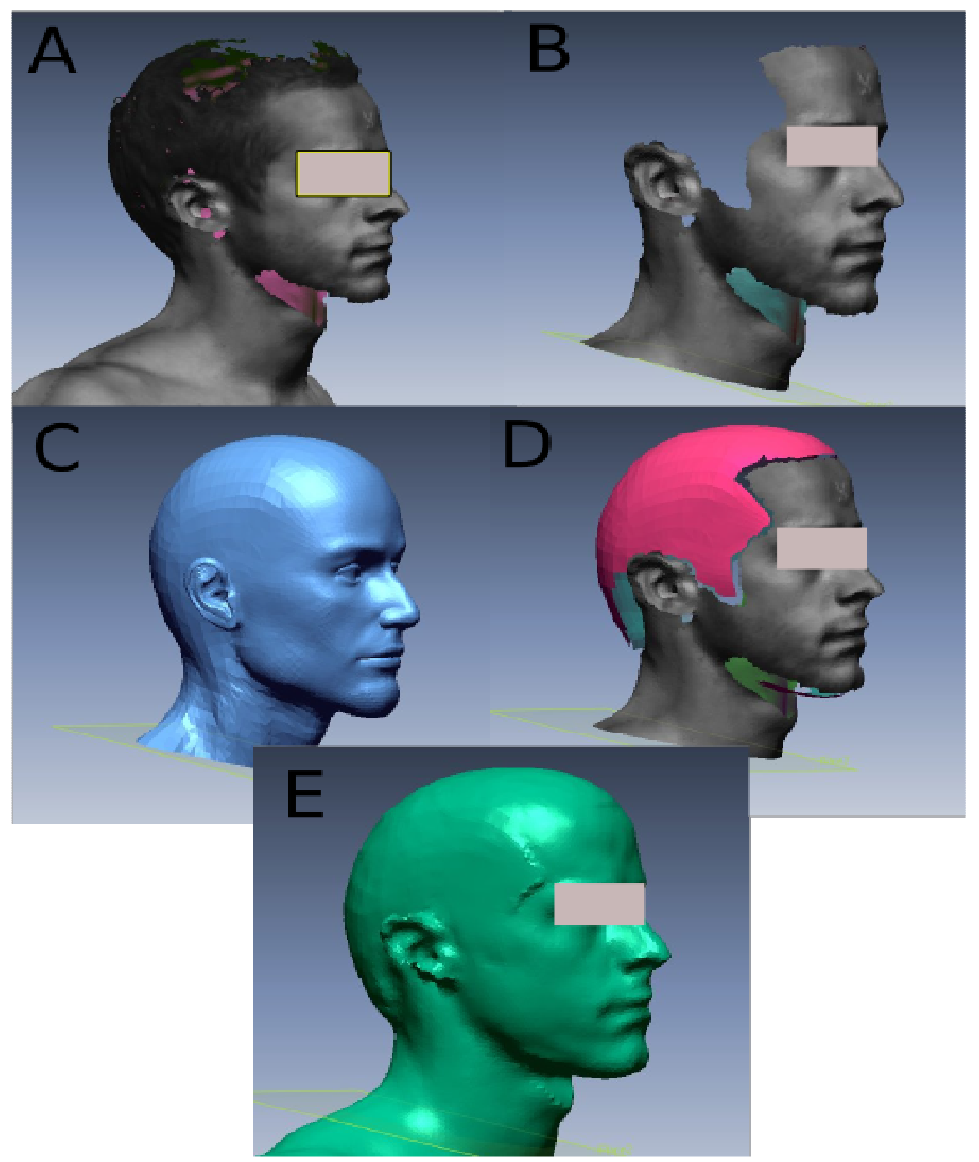

Figure 2: the high resolution interactive hole-filling procedure able to close reasonably large holes like the hair region, not reliably acquired by most scanners. A: original data acquired with a structured light scanner, without using cap. B: segmentation of the reliable regions, presenting large holes. $C$ : The generic model of the part of interest (in this case the head): the region corresponding to the segmented part in $B$ is extracted and used as input for a nonlinear registration procedure. D: After the registration, patches are extracted and zippering is performed. E: the final result. 
Different methods available in literature for hole filling have been analyzed and tested: we adopted as default option the well known volume-based hole-filling method described in [7], based on the computation of distance maps on a discrete grid. The method is fully automatic and is able to cope with large and irregular holes, and the resolution of the mesh obtained can be easily controlled through the discretization step. The mesh is sufficiently good to obtain the automatic skeletonization/partitioning, the detection of salient regions and pose estimation described in the following subsections (e.g. the "raw" processing pipeline). Meshes used for the low-resolution analysis are generated with about ten thousand points and a resolution of about one centimetre.

The result is, however, not very accurate in presence of very large holes, that can appear in the head if the subject does not wear a cap due to reflective hair, or in the armpit region, because the method creates in this case unnatural results.

For the "finer" mesh analysis, if we need to close very large holes in order to apply measurement tools requiring watertight meshes (e.g. to measure volumes) we have therefore developed a different, interactive method.

In this method, while small holes are simply automatically closed with simple approximately planar patches, large holes are handled with a model based approach. The technique is based on a nonrigid registration of a generic model of the body part (e.g. head, as in the example of Fig. 2) over the acquired data and the subsequent merging of the two geometries, preserving the acquired points.

The procedure starts with an interactive cut of the reliable part of the model, and the corresponding segmentation of the generic model. This procedure is currently done with interactive software like Meshlab (we have also tested with success other commercial packages that allows a quick interactive segmentation, like Rapidform XOR Redesign [13]).

A nonlinear spatial transform is computed through a nonlinear registration of the generic model (moving) over the "reliable" acquired data (fixed). The used generic (moving) model derives from the data set distributed as part of the TOSCA project [15].The registration is performed using the method presented in [8]: the deformation model is described by Thin-Plate Splines and the optimization is driven by the "distances" between subsets of the point clouds representing the two corresponding geometries, that are represented as mixtures of Gaussians centered in the meshes nodes. The subsets used as control points are taken from the reliable part of the acquired data (e.g. the face in the case of Fig. 2), and the corresponding region in the reference model. Point density of the sample is not uniform, but it is higher in border region to increase the accuracy of the subsequent mesh zippering,

The computed transformation is then applied to the generic model: from the parts missing in the acquired mesh patches are obtained and a zippering procedure is performed to create the final watertight mesh (Fig. 2).

\section{Low resolution mesh analysis}

The first step of our processing pipeline is then the application of geometric processing algorithms to a low resolution (about 10000 nodes), watertight version of the original human model. The currently applied algorithms are able to recognize salient points, detect pose and segmenting body parts. use

\subsection{Salient point extraction and recognition}

Several methods exists in literature for the extraction of salient points on general meshes, that can be used as well to locate anatomical parts (e.g. eyes, nose, fingers) or articulated junctions. The two main approaches applied for the purpose are the scale-space-based, and the spectral-based methods. Scale-space-based methods exploit perceptual properties of the surface [10] being inspired by the scale theory successfully applied onto the 2D domain. Spectral based methods employ Laplace-Beltrami [11] transform onto 3D surface in order to select points with special properties onto the spectral domain.

We implemented a salient point detector of this kind using the Heat Kernel at a large time to robustly characterize salient points [12]. The Heat Kernel operator $\mathrm{K}_{\mathrm{t}}^{\mathrm{D}}(\mathrm{x}, \mathrm{z})$ represents the solution of the heat diffusion equation on the body surface (domain D) at position $x$ and with initial distribution $\delta(x-z)$. The solution can be easily represented as a function of eigenfunction and eigenvalues of the Laplace-Beltrami operator. 
In [12] it is shown how the Heat kernel with $\mathrm{z}$ equal to $\mathrm{x} \mathrm{K}_{\mathrm{t}}^{\mathrm{D}}(\mathrm{x}, \mathrm{x})$ has extremal points in highly curved areas and that for different values of $t$ the function characterizes at different scales the surface features. This means that it is possible to detect locally salient points searching for maxima of the functions at fixed $t$ and to identify different features sampling the function at different values of $t$, building the so-called "Heat Kernel Signature" (HKS).

Using local saliency and HKS it is possible to find a lot of characteristic points and try to recognize a large number of anatomical landmarks. However, the recognition of all these points (e.g. with a Nearest Neighbor search over a set of labeled point templates) would be not trivial.

As stated before our approach is to have a hierarchical analysis with an initially raw characterization of the shape and then a refined description using the previously extracted context information. This means that we could try to have first a more simple and robust recognition of a few rough anatomical landmarks and then use a context aware method for the subsequent high resolution analysis.

To do this, we apply the Heat Kernel analysis, using a limited number of eigenvalues and for time exceeding a large threshold, we extract a few local maxima of $\mathrm{K}_{\mathrm{t}}^{\mathrm{D}}(\mathrm{x}, \mathrm{x})$ representing not very "local" features, but points that are well characterized at a global scale. For a sufficient large $t$ we found that all the points detected in a training set of 12 models were successfully recognized sing the Heat Kernel Signature as head, hand finger, foot finger and no salient points in different positions have been detected.

We use this small number of reliably labeled salient point as a basis for the hierarchical analysis: they can be used as input for the skeleton-based analysis and stick figure optimization, but we also plan to use them to obtain context-aware heat kernel based signatures for the reliable recognition of other salient points, detected with the same method, but with decreasing values of $t$ (and a larger number of eigenvalues of the Laplace-Beltrami operator) in order to capture more local features.

\subsection{Skeletonization and partitioning}

Given a low resolution and watertight representation of the body, we apply a procedure to obtain a pose estimation and rough segmentation of the mesh through the extraction and labeling of the curve skeleton of the mesh and an optimization step finding a symmetric and reasonable stick figure adapted to the skeleton.

The procedure has been described in [9] and consists of extracting a curve skeleton of the mesh with an efficient method based on voxel coding and active contours. Starting from the head salient point extracted on the surface we can compute the shortest path from the farthest inner point and the selected point. Other branches connecting the first one to the farthest points are then similarly created, generating a rough skeletal tree. These branches are finally centered with an optimization procedure driven by the gradient of the distance from border map and a gradient vector flow based force related to the external surface normals. During the optimization steps, automatic removal of duplicated or superimposed parts is performed. The algorithm is fast and reliable if the topology of the mesh is sphere-like. The algorithm is able to compute a limited number of external branches of the tree, number that is finally reduced to five with a simple cleaning procedure removing smaller leaves of the skeletal tree.

With simple heuristics based on skeletal branches lengths and directions, and a consistency check related to the extracted surface salient points, it is possible to recognize head, trunk and limbs from the skeleton (see [9] for details). Associating then scalar values on the skeleton points (e.g. diameter, eccentricity, angle) it is possible to detect features (skeletal salient points) corresponding to joints and have a rough segmentation of the mesh, that can be propagated to the body volume and to the different meshes (original, watertight and low resolution).

\subsection{Pose estimation}

A rough pose estimation is finally obtained with an optimization procedure minimizing a cost function forcing symmetry and using a priori information.

Skeletal and surface salient points are used to initialize the stick figure representing the axes of head neck, trunk and arms with related joints. The stick figure is then refined with an optimization procedure, performed with nonlinear leas squares method, moving joints coordinates from the original position in order to minimize an objective function depending on distance of stick segments from curve skeleton and features, with additional terms forcing symmetry and similarity to average body proportions. In this way it is possible to improve the accuracy of the skeleton based body parts segmentation that is caused by the low quality of the hole filling. 
The stick figure provides directly an estimation of arms and trunk sizes and is able to give the correct orientation of the body parts necessary to obtain accurate interactive evaluation of diameters and other distances.

\subsection{Low-resolution processing results}

Fig. 3 shows an example of the results of the three low resolution processing modules described. The left image shows the curve skeleton (the color encodes the distance from border value) and the labeled salient points extracted (green=hand, red=head, blue=foot). The right image show the stick figure encoding the pose and the limb lengths, with recognition of the different body parts.

As seen before, the automatic identification and labeling of salient points correctly worked on all the 12 meshes tested, identifying always at least an extremal point for each limb. The centerline extraction and segmentation correctly identified the different body parts in all the 12 models tested. The accuracy of the skeleton-based segmentation is not always very high due to the low-resolution hole filling, creating anomalous structures around armpits and crotch. More correct joint locations are found through the stick figure, and the accuracy of the body segments lengths and diameters is going to be validated as well as the other measurements interactively performed, on a wider set of heterogeneous body scanner acquisitions.
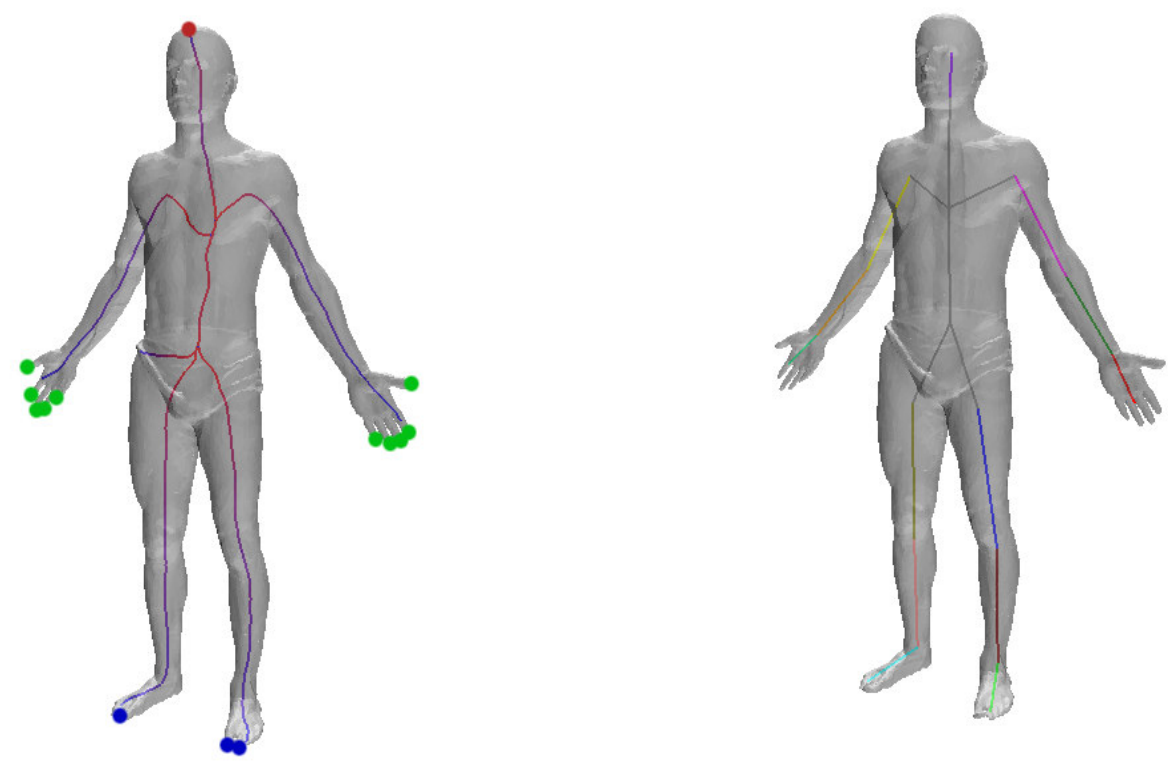

Figure 3: Left: 10Kpoints mesh with automatically extracted labeled points on the surface and curve-skeleton. Right: pose estimation with the computed and labeled stick figure with recognized body parts.

\section{Application: measuring anthropometric parameters}

The work described is done in a collaboration between the departments of Computer Science and Motor and sport Science of the University of Verona, currently being extended to other computer science and health care research center across Europe. The aim of the research is to compute and validate morphological parameters from $3 \mathrm{D}$ models acquired with different scanners and to correlate the results with different metabolic indicators. User interfaces enabling the operator to perform and collect different computer assisted measurements on original 3D models exploiting also information derived from the previously described "low resolution" analysis have been realized and partially tested $[14,16]$. Examples of interactive measurements are shown in Fig 4.

The measurement set implemented tries to simulate the manual measurement currently performed in classical anthropometric research and validation is performed comparing the values of computer assisted and tape-based measurements. Preliminary encouraging results are reported in $[14,16]$ and more accurate validation tests are currently being performed on a larger set of individuals. 

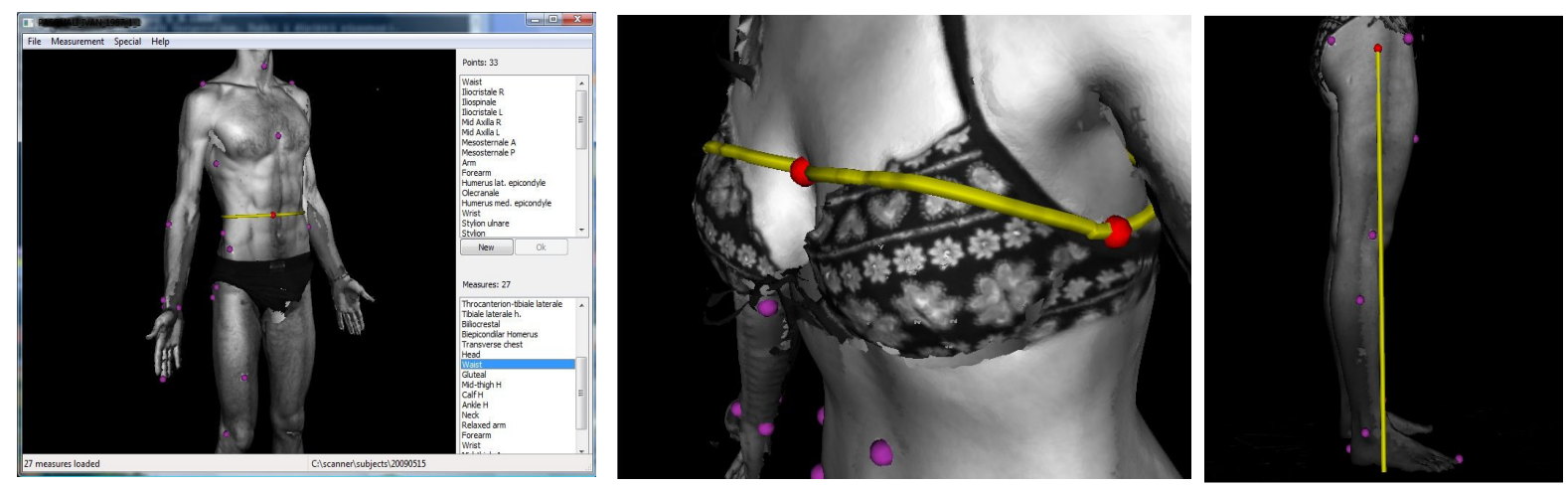

Figure 4: user interface for interactive measurements and examples of standard measurements as girths and lengths, that are related to geodesic paths and euclidean distances between landmarks.

It must, however, noted that this kind of validation is not always reliable especially for the fact that tape-based measurements that should be compared with the electronic ones often involve complex procedures and body deformation.

Automatic processing is also able to provide directly some measurements like volumes (of full body and subparts), lengths, maximal girths. These values have shown to be almost pose-independent [14] and clearly can encode relevant information on body shape.

Other validation tests that are planned will evaluate the influence of different pre-processing and hole filling methods on the accuracy of volume and geodesic paths length and, as soon as the finer location of anatomical landmarks performed with the Heat Kernel Signature will be realized, another set of tests aimed at checking the possibility of recognizing classical manually placed landmarks will be performed.

\section{Discussion}

Digital anthropometry is going to relevantly improve the possibility of analyzing the human body shape and its evolution. Structured light and laser based 3D body scanners can, in fact, acquire non invasively and in a few seconds the detailed shape of the body surface with a point accuracy of about 1 millimeter. Several commercial applications of this technology have been already developed, especially related to apparel industry and ergonomics.

In order to store and exploit maximally the information captured by these devices, especially for medical applications, it would be important to define some standard or good practices for the handling of the data created.

Complete meshes with associated metadata related to the scanning procedure should be collected instead of simple sets of measurements: something like a DICOM object model for storing body models should be defined and used by manufacturers to ensure interoperability.

Measurements and shape analysis methods applied to derive anthropometric parameters should also be standardized in some way in order to increase the possibility of performing large scale surveys or statistical studies. The use of well defined methods and open-source tools to process the captured geometries is, in our opinion a good choice in order to increase collaboration in anthropometric research. Another exciting opportunity related to digital anthropometry is the possibility of not limiting the body shape analysis to a limited set of lengths, girths or volumes. Digital models of the human body provided by body scanners contain much more information and statistical methods like principal component analysis could be used to define particular deformation of the body or of body subparts that could be correlated with well known metabolic indicators.

A lot of work seem necessary to fully exploit the potential of 3D scanning technology in anthropometric studies.

In this paper we presented our contribution towards this goal, i.e. a work in progress that aims at defining a processing pipeline for body scanner data processing and at applying recent advances in geometry processing algorithms to derive relevant morphological parameters. 


\section{References}

1. Wells JC, Cole TJ and Treleaven P Age-variability in body shape associated with excess weight: the UK national sizing survey. Obesity, 435-441, 2008

2. Wells JC, Cole TJ, Bruner D, and Treleaven P. Body shape in American and British adults: between-country and inter-ethnic comparisons. Int J Obes., pages 152-159, 2008

3. Wang J, Gallagher D, Thornton JC, Yu W, Horlick M, and Pi-Sunyer FX. Validation of a 3-dimensional photonic scanner for the measurement of body volumes, dimensions and percentage body fat. Am J Clin Nutr, pages 809-816, 2006.

4. Wells JC, Cole TJ, Bruner D, and Treleaven P. Body shape in American and British adults: between-country and inter-ethnic comparisons. Int J Obes., pages 152-159,

5. Lin JD, Chiou WK, Weng HF, Fang JT, and Liu TH. Application of three-dimensional body scanner: observation of prevalence of metabolic syndrome. Clin Nutr., pages 1313-23, 2004.

6. Karia P. Simmons. Body measurement techniques: a comparison of three-dimensional scanning and physical anthropometric methods. Ph.D dissertation, North Carolina State University, 2001.

7. Ju, T. Robust Repair of Polygonal Models. Proceedings of ACM SIGGRAPH, 2004 ACM Transactions on Graphics, 23(3):888-895

8. Bing Jian, Baba C. Vemuri, A Robust Algorithm for Point Set Registration Using Mixture of Gaussians, Tenth IEEE International Conference on Computer Vision (ICCV'05) Volume 2, pp.1246-1251, 2005

9. C. Lovato, U. Castellani and A.Giachetti. Automatic segmentation of scanned human body using curve skeleton analysis. In A. Gagalowicz and W. Philips (Eds.): MIRAGE 2009, LNCS 5496, Springer, pp.34-45

10. Andrei Zaharescu, Edmond Boyer, Kiran Varanasi, and Radu P. Horaud. Surface feature detection and description with applications to mesh matching, Proceedings of the IEEE Conference on Computer Vision and Pattern Recognition (Miami Beach, Florida), 2009.

11. Levy, B. 2006. Laplace-Beltrami Eigenfunctions Towards an Algorithm That "Understands" Geometry. In Proceedings of the IEEE international Conference on Shape modelling and Applications 2006 (June 14 - 16, 2006). Shape modelling International.

12. Sun J., Ovsjanikov M., Guibas L.: A concise and provably informative multi-scale signature based on heat diffusion. In Eurographics Symposium on Geometry Processing (SGP) (2009).

13. Rapidform web site (accessed 2010) http://www.rapidform.com

14. C. Lovato, U. Castellani, S. Fantoni, C. Milanese, C. Zancanaro, A. Giachetti, Computer assisted estimation of anthropometric parameters from whole body scanner data in N. Magnenat-Thalmann (Ed.): Modelling the Physiological Human - Proc. 3DPH 2009 pp.71-83 LNCS 5903, 71-83, December 2009.

15. A. M. Bronstein, M. M. Bronstein, R. Kimmel. Numerical geometry of non-rigid shapes, Springer, 2008. Dataset web address: http://tosca.cs.technion.ac.il/book/resources_data.html

16. C. Lovato, C Milanese, A. Giachetti, C. Zancanaro. Three-dimensional digital anthropometry using the BodyScan 3D Body Scanning Technologies. Lugano, Switzerland, 19-20 October 2010

17. P. Cignoni, M. Callieri, M. Corsini, M. Dellepiane, F. Ganovelli, G. Ranzuglia. MeshLab: an Open-Source Mesh Processing Tool. Sixth Eurographics Italian Chapter Conference, page $129-136-2008$

18. Meshlab web site (accessed 2010) http://meshlab.sourceforge.net/ 